\title{
Evaluation of association studies, a systematic review and meta-analysis of CYP1A1 polymorphisms, and colorectal cancer risk
}

\author{
Lingjun $\mathrm{Xu}^{1}$, Xiaofeng $\mathrm{He}^{2}$, and Xiaoping Wang ${ }^{1}$ \\ ${ }^{1}$ Jinan University \\ ${ }^{2}$ Changzhi Medical College Affiliated Heping Hospital
}

November 9, 2020

\begin{abstract}
Backgroud: 37 publications and seven previous meta-analyses have been reported to investigate the correlation between CYP1A1 T3801C and A2455G polymorphisms with CRC risk. However, the results were contradictory. Original studies were not involved in previously published meta-analyses. Moreover, their meta-analyses did not evaluate positive results to identify multiple comparisons. Objectives: We performed an updated meta-analysis and systematically evaluated the published meta-analyses on the association between CYP1A1 T3801C and A2455G polymorphism with CRC susceptibility. Results: CYP1A1 T3801C polymorphism was not associated with CRC risk when all the selected studies were merged. However, subgroup analyses according to ethnicity, geographic region, location of CRC, gender and smoking, showed a significant association in rectal cancer and females. However, after BFDP correction, false significant associations were observed in rectal cancer (CC vs. TT: $\mathrm{BFDP}=0.998 ; \mathrm{CC}$ vs. $(\mathrm{TT}+\mathrm{TC}): \mathrm{BFDP}=0.998 ; \mathrm{C}$ vs. $\mathrm{T}: \mathrm{BFDP}=0.999)$ and females $(\mathrm{TC}$ vs. $\mathrm{TT}: \mathrm{BFDP}=0.999)$. A significantly increased CRC risk was yielded in overall analysis, Caucasians, Europe countries populations, and males for CYP1A1 A2455G polymorphism. After BFDP correction, associations still be significant only in Europe countries populations $(\mathrm{AG}$ vs. $\mathrm{AA}: \mathrm{BFDP}=0.680 ;(\mathrm{AG}+\mathrm{GG})$ vs. $\mathrm{AA}: \mathrm{BFDP}=0.475 ; \mathrm{G}$ vs. $\mathrm{A}: \mathrm{BFDP}=0.126)$. However, when we further performed a sensitivity analysis and BFDP test, false significant associations were observed in all analyses (BFDP $>0.8$ ). Conclusions: In summary, this study suggests that these positive findings may result from false-positive results, rather than from true associations or biological factors.
\end{abstract}

Evaluation of association studies, a systematic review and meta-analysis of $C Y P 1 A 1$ polymorphisms, and colorectal cancer risk

\section{Lingjun $\mathrm{Xu}^{1}$, Xiaofeng $\mathrm{He}^{2}$, Xiaoping Wang ${ }^{1}$}

${ }^{1}$ Department of Pain Management, The First Affiliated Hospital, Jinan University, Guangzhou, 510632, China;

2 Department of Institute of Evidence-based medicine, Heping Hospital Affiliated to Changzhi Medical College, Shanxi, Changzhi, 046000, China

Correspondence: Xiaoping Wang (txp2938@jnu.edu.cn)

\section{Abstract}

Background: 37 publications and seven previous meta-analyses have been reported to investigate the correlation between CYP1A1 T3801C and A2455G polymorphisms with CRC risk. However, the results were contradictory. Original studies were not involved in previously published meta-analyses. Moreover, their meta-analyses did not evaluate positive results to identify multiple comparisons. 
Objectives: We performed an updated meta-analysis and systematically evaluated the published metaanalyses on the association between CYP1A1 T3801C and A2455G polymorphism with CRC susceptibility.

Results: CYP1A1 T3801C polymorphism was not associated with CRC risk when all the selected studies were merged. However, subgroup analyses according to ethnicity, geographic region, location of CRC, gender and smoking, showed a significant association in rectal cancer and females. However, after BFDP correction, false significant associations were observed in rectal cancer $(\mathrm{CC}$ vs. TT: $\mathrm{BFDP}=0.998 ; \mathrm{CC}$ vs. $(\mathrm{TT}+\mathrm{TC})$ : $\mathrm{BFDP}=0.998 ; \mathrm{C}$ vs. $\mathrm{T}: \mathrm{BFDP}=0.999)$ and females $(\mathrm{TC}$ vs. TT: $\mathrm{BFDP}=0.999)$. A significantly increased CRC risk was yielded in overall analysis, Caucasians, Europe countries populations, and males for CYP1A1 A2455G polymorphism. After BFDP correction, associations still be significant only in Europe countries populations ( $\mathrm{AG}$ vs. $\mathrm{AA}: \mathrm{BFDP}=0.680 ;(\mathrm{AG}+\mathrm{GG})$ vs. $\mathrm{AA}: \mathrm{BFDP}=0.475 ; \mathrm{G}$ vs. $\mathrm{A}: \mathrm{BFDP}=0.126)$. However, when we further performed a sensitivity analysis and BFDP test, false significant associations were observed in all analyses (BFDP > 0.8).

Conclusions: In summary, this study suggests that these positive findings may result from false-positive results, rather than from true associations or biological factors.

Keywords: CYP1A1, polymorphism, colorectal cancer, meta-analysis

\section{Introduction}

Colorectal cancer (CRC) has been an public health problem for many years because of increasing incidence and high mortality rate despite some overall improvements in diagnosis and treatment $[1,2]$. It is a common form of cancer, with more than 1.5 million new patients diagnosed every year worldwide [3] and more common in developed than developing countries [4]. Previously published studies have indicated that age over 60 years, alcohol consumption and tobacco habits may contribute to sporadic CRC risk [5, 6]. Moreover, the development of CRC is widely considered as a multi-step, multi-factorial process involving gene-gene and gene-environment interactions [7]. It has been suggested that inter-individual differences including single nucleotide polymorphism (SNP) might influence CRC risk $[8,9]$.

The cytochrome P450 enzymes (CYPs) take part in the metabolism of carcinogenic compounds in the human body [10]. CYP1A1 is expressed in the large bowel [11]. Because of the involvement of CYP1A1 in the metabolism of PAHs and estrogens, and perhaps cruciferous vegetables and heterocyclic amines [12], it might be expected that CYP1A1 variants might influence the risk of colorectal cancer. A commonly studied SNP in the CYP1A1 gene has been indicated to associate with CRC risk, which was localized on chromosome 15q22 [13]. The commonly reported the $3801 \mathrm{~T}>\mathrm{C}$ (also referred to as $2 \mathrm{~A}, \mathrm{~m} 1$, or rs4646903) and A2455G polymorphisms (also known as E7, ${ }^{*} 2 \mathrm{C}, \mathrm{m} 2$, rs1048943) with CRC risk [14]. The T3801C and A2455G polymorphisms might change the level of gene expression or messenger RNA stability, resulting in a highly inducible activity of the enzyme [15].

To date, 37 publications (supplemental Table 2) and seven previous meta-analyses [16-22] have been reported to investigate the correlation between the CYP1A1 T3801C and A2455G polymorphisms with CRC risk. However, the results were inconsistent or even contradictory. In addition, a lot of original studies did not involve in previously published meta-analyses (supplemental Table 1). Moreover, previously published metaanalyses also did not evaluate positive results to identify multiple comparisons. Therefore, we performed an updated meta-analysis and systematically evaluated the published meta-analyses on the association between CYP1A1 T3801C and A2455G polymorphism with CRC susceptibility.

\section{Materials and Methods}

The present study was performed according to the Preferred Reporting Items for Systematic Reviews and Meta-Analyses guideline [23].

\section{Search strategy}

We systematically searched the PubMed, Embase, China National Knowledge Infrastructure (CNKI), and Wan Fang databases (up to Sep 12, 2020) by using the following strategy: ('CYP1A1 OR 'Cytochrome 
P450 1A1') AND ('polymorphism' OR 'variant' OR 'variation' OR 'mutation' OR 'SNP' OR 'genome-wide association study' OR 'genetic association study' OR 'genotype' OR 'allele') AND ('colorectal' OR 'colon' OR 'rectal'). The language was not limited in this study. In addition, the reference lists was checked if the publications were relevant review and meta-analyses studies.

\section{Inclusion and exclusion criteria}

The inclusion criteria were as follows: (1) case-control or cohort studies, (2) explored the correlation between the CYP1A1 T3801C and A2455G polymorphisms and CRC risk, and (3) gave the genotype data of cases and controls or the odds ratio (OR) with their 95\% confidence intervals (CI). Exclusion criteria were as follows: (1) review articles, (2) case reports, (3) editorials, (4) systematic review and meta-analyses, (5) other SNP, (6) other disease, (7) animal studies, and (8) duplicate data.

\section{Data extraction and quality score assessment}

Data was extracted and checked by two investigators independently. Disagreement was settled through discussion and consensus. The following information was collected: (1) first author name, (2) year of publication, (3) country, (4) geographic region, (5) ethnicity, (6) sample size, (7) source of controls, (8) type of control, (9) matching, (10) genotypes distribution, (11) location of CRC, (12) site of CRC, and (12) gender.

Scale for quality assessment criteria was shown in Table 1. All eligible studies quality was also independently assessed by two authors. Total scores ranged from 0 (worst) to 21 (best). If scores were $<13$ (the eligible studies were regarded as low quality) and If scores of [?] 13 (the eligible studies were regarded as high quality).

\section{Statistical analysis}

Crude ORs and 95\% CIs was applied to assess the association between the CYP1A1 T3801C and A2455G polymorphism with CRC risk. The following genetic models were used: a dominant model (CYP1A1T3801C: $(\mathrm{TC}+\mathrm{CC})$ vs. TT and CYP1A1 A2455G: $(\mathrm{AG}+\mathrm{GG})$ vs. AA), a recessive model $(C Y P 1 A 1$ T3801C: CC vs. (TT $+\mathrm{TC})$ and CYP1A1 A2455G: GG vs. (AA + AG), an additive model (CYP1A1T3801C: TC vs. TT and CC vs. TT; $C Y P 1 A 1$ A2455G: AG vs. AA and GG vs. AA), and an allelic model (CYP1A1 T3801C: $\mathrm{V}$ vs. T and CYP1A1 A2455G: G vs. A).

Heterogeneity among studies was assessed to use $P_{\text {heterogeneity }}\left(P_{\mathrm{h}}\right)$ and $I^{2}$ values [24]. A fixed-effects model (Mantel-Haenszel method) [25] was considered if $P$ [?] 0.10 and/or $I^{2}$ [?] 50\%; otherwise, a randomeffects model (DerSimonian and Laird method) was regarded [26]. Subgroup analyses were calculated by ethnicity, geographic region, gender, location of CRC, and site of CRC. Sensitivity analyses were conducted to investigate the stability of results. The following two methods were considered: (1) One by one exclusion and (2) a data set was built only selecting high-quality and controls of Hardy-Weinberg equilibrium (HWE) studies. HWE was tested by a Chi-square goodness-of-fit test. If $P<0.05$, the controls were considered as Hardy-Weinberg disequilibrium (HWD). Begg's funnel plot [27] and Egger's regression asymmetry test [28] were employed to assess publication bias. A nonparametric "trim and fill" method [29] was selected if a obvious publication bias was observed. In addition, a meta-regression analysis was employed to explore the sources of heterogeneity among studies. Furthermore, a Bayesian false discovery probability (BFDP) was used to assess the false positive results [30]. A cutoff value of BRDP was set up to be a level of 0.8 and a prior probability of 0.001 to evaluate whether the significant associations were the false positive. All statistical analyses were calculated applying Stata 12.0 software (STATA Corporation, College Station, TX).

\section{Results}

\section{Study characteristics}

A flow diagram was shown in Fig. 1. Overall, 37 publications were selected to analyze the CYP1A1 T3801C and A2455G polymorphisms with CRC risk. The current and published meta-analyses involving studies were shown in supplemental Table 1. The eligible studies characteristics were listed in supplemental Table 2. Of 
these, 27 studies (involving 9,672 cases and 12,689 controls; eight studies on Asians, fourteen on Caucasians, and five on mixed populations; ten studies on Asia; nine on Europe; five on North America, one on South America, and one on Ocean) for CYP1A1 T3801C polymorphism and 24 studies (involving 8,933 cases and 11,692 controls; seven studies on Asians, twelve on Caucasians, and five on mixed populations; seven studies on Asia; nine on Europe; five on North America, two on South America, and one on Ocean) for CYP1A1 A2455G polymorphism were performed in the current pooled analysis. Supplementary Tables 3-5 shows the study characteristics by location of CRC, gender, and site of CRC, respectively.

\section{Quantitative synthesis}

\section{The $C Y P 1 A 1$ T3801C polymorphism and CRC risk}

Overall, the CYP1A1 T3801C polymorphism was not associated with CRC risk when all the selected studies were merged, as shown in Table 2. Then, subgroup analyses according to ethnicity, geographic region, location of CRC, gender, and smoking, a significant association was only observed in rectal cancer (CC vs. TT: $\mathrm{OR}=0.73,95 \%$ CI: $0.55-0.97$; $\mathrm{CC}$ vs. $(\mathrm{TT}+\mathrm{TC}): \mathrm{OR}=0.75,95 \%$ CI: $0.57-0.98 ; \mathrm{C}$ vs. $\mathrm{T}: \mathrm{OR}=$ 0.89, 95\% CI: $0.80-1.00$ ) and females (TC vs. TT: OR $=1.27,95 \%$ CI: $1.00-1.62$ ). However, after BFDP correction, associations did not be observed in rectal cancer (CC vs. TT: BFDP $=0.998$; $\mathrm{CC}$ vs. $(\mathrm{TT}+$ $\mathrm{TC}): \mathrm{BFDP}=0.998 ; \mathrm{C}$ vs. $\mathrm{T}: \mathrm{BFDP}=0.999)$ and females $(\mathrm{TC}$ vs. $\mathrm{TT}: \mathrm{BFDP}=0.999)$.

\section{The CYP1A1 A2455G polymorphism and CRC risk}

Tables 3 shows the pooled results on the association between the CYP1A1 A2455G polymorphism and CRC risk. A significantly increased $\mathrm{CRC}$ risk was yielded in overall analysis (GG vs. AA: $\mathrm{OR}=1.39,95 \%$ CI: $1.03-1.88$; GG vs. $(\mathrm{AG}+\mathrm{AA}): \mathrm{OR}=1.32,95 \% \mathrm{CI}: 1.10-1.57)$. Then, Subgroups were conducted by ethnicity, geographic region, location of CRC, gender, and smoking. A significantly increased CRC risk was also found in Caucasians (AG vs. AA: OR $=1.26,95 \%$ CI: $1.10-1.46$; GG vs. AA: OR $=1.71,95 \%$ CI: 1.00-2.93; $(\mathrm{AG}+\mathrm{GG})$ vs. $\mathrm{AA}: \mathrm{OR}=1.27,95 \%$ CI: $1.04-1.54 ; \mathrm{G}$ vs. $\mathrm{A}: \mathrm{OR}=1.27,95 \%$ CI: $1.12-1.43)$, Europe countries populations (AG vs. AA: OR $=1.43,95 \%$ CI: $1.20-1.70 ;(\mathrm{AG}+\mathrm{GG})$ vs. AA: OR $=1.45$, 95\% CI: $1.23-1.72$; $\mathrm{G}$ vs. A: $\mathrm{OR}=1.43,95 \%$ CI: $1.22-1.66)$, and males $((\mathrm{AG}+\mathrm{GG})$ vs. $\mathrm{AA}: \mathrm{OR}=1.47$, 95\% CI: 1.12-1.94). after BFDP correction, association was still significant in Europe countries populations $(\mathrm{AG}$ vs. $\mathrm{AA}: \mathrm{BFDP}=0.680 ;(\mathrm{AG}+\mathrm{GG})$ vs. $\mathrm{AA}: \mathrm{BFDP}=0.475 ; \mathrm{G}$ vs. $\mathrm{A}: \mathrm{BFDP}=0.126)$.

\section{Heterogeneity and sensitivity analyses}

Significant heterogeneity was observed, as shown in Tables 2 and 3. We observed that ethnicity, HWE, and quality score were not source of source of heterogeneity. However, when we excluded Pereira Serafim PV et al. (supplemental Table 1), $I^{2}$ value dropped and significant association was found in overall analysis ( $\mathrm{G}$ vs. A: $\mathrm{OR}=1.15,95 \% \mathrm{CI}: 1.02-1.30$ ) for $C Y P 1 A 1$ A2455G polymorphism. Furthermore, after BFDP correction, no significant association was observed in $\mathrm{G}$ vs. A $(\mathrm{BFDP}=0.998)$

Sensitivity analysis was conducted using two methods. First, the results did not change when we applied one by one exclusion in the present study. Further, when we only involved high-quality and HWE in controls studies, a significant association was yielded in Asians (C vs. T: OR $=0.92,95 \%$ CI: $0.85-1.00$ ), Asia countries populations (C vs. T: $\mathrm{OR}=0.92,95 \%$ CI: $0.85-1.00)$, rectal cancer $(\mathrm{CC}$ vs. $\mathrm{TT}$ : $\mathrm{OR}=0.73,95 \%$ CI: $0.55-0.97$; $\mathrm{CC}$ vs. (TT $+\mathrm{TC}$ ): $\mathrm{OR}=0.75,95 \%$ CI: $0.57-0.98$; $\mathrm{C}$ vs. $\mathrm{T}: \mathrm{OR}=0.89,95 \%$ CI: $0.80-1.00)$, and females (TC vs. TT: OR $=1.27,95 \% \mathrm{CI}: 1.00-1.62$ ) for the CYP1A1 T3801C polymorphism, as also shown in Table 2; concerning the CYP1A1 T2455G polymorphism, a significant association was observed in overall analysis, Asians, Caucasians, Asia countries populations, Europe countries populations, and males, as also shown in Table 3. However, after BFDP correction, no significant associations were found in the above all analyses (all BFDP >0.8), as also listed in Tables 2-3.

\section{Publication bias}

No obvious publication bias was found on the CYP1A1 A2455G polymorphism with CRC risk. However, a significant publication bias was observed on the CYP1A1 T3801C (C vs. T: $P=0.013$ ) polymorphism 
with CRC risk. Figure 2 shows the non-parametric 'trim and fill' method funnel plot. The result did not revised in overall analysis ( $\mathrm{C}$ vs. $\mathrm{T}$ : $\mathrm{OR}=0.94,95 \% \mathrm{CI}$ : $0.84-1.04)$ indicating add studies can not affect the merging result.

\section{Discussion}

Hayashi S et al. In 1992 (Supplemental Table 1) first reported an association on the CYP1A1 T3801C and A2455G polymorphisms and with CRC risk. So far, a lot of studies have performed the above two issues. However, the results are still inconsistent. Moreover, seven previously published meta-analyses [16- 22] have arrived at significant disagreement results, especially in different races, as list in supplemental Tables 6 and 7. Moreover, As far as we know that this is the first meta-analysis to investigate the significant results by BFDP test to avoid confounding factors.

The CYP1A1 T3801C polymorphism was associated with rectal cancer risk in overall races and CRC risk in females; the CYP1A1 A2455G polymorphism was associated with an increased CRC risk in overall analysis, Caucasians, Europe countries populations, and males. Five genetic model and several subgroup analyses was conducted, this analysis came at the cost of multiple comparisons, in this instance, the pooled OR with their 95\% CI must be adjusted to avoid the false positive results [32]. Wakefield et al. [31] provided a precise Bayesian measure of false discovery to adjust the positive results. The method especially suited for SNP and disease susceptibility study. Therefore, we applied BFDP value to evaluate the positive results in this study. After we further performed a sensitivity analysis and BFDP test, BFDP $>0.8$ were found in all analyses.

Therefore, these positive associations might most likely result from false-positive results, rather than from true associations or biological factors. The reason may be because some small sample and low-quality studies were easier to accept if there was a positive report. They tend to yield false-positive results because they may be not rigorous and are often of low-quality. In addition, controls of HWD may be genotype errors and selection bias in molecular epidemiological studies.

Therefore, we performed a sensitivity analysis restricted to studies that only included high-quality articles and controls of HWE.

Supplemental Tables 6- 7 list the results of seven previously published meta-analyses [16-22] on the $C Y P 1 A 1$ T3801C and A2455G polymorphisms with CRC risk, respectively. The largest sample size study [19] was performed in 2014 involving 18 studies (7,171 cases and 8,957 controls) for the CYP1A1 T3801C polymorphism; on the CYP1A1 A2455G polymorphism, The largest sample size study [16] was reported in 2016 including 20 studies (8665 cases and 8853 controls). Several studies should be excluded in the above two studies [16, 29]. The present study included 27 studies $(9,672$ cases and 12,689 controls) on the CYP1A1 T3801C polymorphism and 24 studies (8,933 cases and 11,692 controls) on the CYP1A1 A2455G polymorphism with CRC risk. In addition, four previous meta-analyses $[16,18,19,21]$ reported the association on the CYP1A1T3801C polymorphism with CRC risk, only a meta-analysis [18] observed a significant decreased CRC risk in Chinese population; another three meta-analyses found that the CYP1A1 T3801C polymorphism was not significantly associated with CRC risk; five published meta-analyses [16, 17, 20-22] have been performed to investigate the association on the CYP1A1 A2455G polymorphism with CRC risk. Of these, four studies [16, 20-22] reported a significantly increased CRC risk in Asians and Caucasians and one study [17] observed a significantly increased CRC risk in Asia and Europe, but not in America. These results of previously published studies were not consistent, especially in ethnicity. An obvious inconsistency was found in classification of ethnic groups among these published meta-analyses, cells with red color in supplemental Table 1. Moreover, we still found a significant difference on the HWE of controls in these published studies (cells with blue color in supplemental Table 1). In addition, previously published meta-analyses involved incomplete studies and some repeat studies. All studies did not adjusted significant results for multiple comparisons. Therefore, their meta-analyses are still incredible.

Though all our efforts were made to improve our research, the current study still exist several limitations. First, only published articles were included while positive results are known to be published more readily than negative ones. Second, several subgroup analyses only included small sample size, such as in smoking 
subgroups (Table 2 and Table 3), the results should be interpreted with caution and it is necessary that a well-designed large sample study to explore the true association. Third, we did not collect the adjusted data because the original study provided little information. Hence, a more precise analysis should be performed when enough data was available in future.

In summary, this study suggests that these positive findings may most likely result from false-positive results, rather than from true associations or biological factors.

\section{Competing Interests}

The authors declare that there are no competing interests associated with the manuscript.

\section{Funding}

None.

\section{Author Contribution}

L.X. was involved in the conception and design of the meta-analysis. X.H. contributed to study selection, data extraction, statistical analysis and writing of manuscript. L.X. and X.H. contributed to the preparation of the meta-analysis and literature review. X.W. critically revised the manuscript and all authors read and approved the manuscript.

\section{Reference}

1. Heavey PM, McKenna D, Rowland IR (2004). Colorectal cancer and relationship between genes and the environment. Nutr Cancer, 48, 124-41.

2. Parkin DM, Bray F, Ferlay J, et al (2005). Global cancer statistics 2002. CA Cancer J Clin, 55, 74-108.

3. Brenner H, Kloor M, Pox CP. Colorectal cancer. Lancet. 2014 383:1490-1502.

4. Weitz J, Koch M, Debus J, Hohler T, Galle PR et al (2005) Colorectal cancer. Lancet 365:153-165.

5. INCA (Instituto Nacional do Câncer). [Internet]. Accessed April 9, 2015. Available at [http://www.inca.gov.br].

6. Khan N, Afaq F, Mukhtar H. Lifestyle as risk factor for cancer: Evidence from human studies. Cancer Lett. 2010;293:133-143.

7. Ahmed FE. Gene-gene, gene-environment \& multiple interactions in colorectal cancer. J Environ Sci Health C Environ Carcinog Ecotoxicol Rev. 2006; 24:1-101.

8. Tomlinson IP, Webb E, Carvajal-Carmona L, Broderick P, Howarth K, Pittman AM, Spain S, Lubbe $\mathrm{S}$, Walther A, et al. A genome-wide association study identifies colorectal cancer susceptibility loci on chromosomes 10p14 and 8q23.3. Nat Genet 2008; 40: 623-630

9. Abulí A, Bessa X, González JR, Ruiz-Ponte C, Cáceres A, Muñoz J, Gonzalo V, Balaguer F, FernándezRozadilla C, González D, et al. Susceptibility genetic variants associated with colorectal cancer risk correlate with cancer phenotype. Gastroenterology 2010; 139: 788-796, 796.e1-e6

10. Lewis DF. P450 structures and oxidative metabolism of xenobiotics. Pharmacogenomics 4: 387-395, 2003.

11. McKay JA, Murray GI, Weaver RJ, Ewen SW, Melvin WT, Burke MD. Xenobiotic metabolising enzyme expression in colonic neoplasia. Gut 1993;34:1234-9.

12. Hummerich J, Zohm C, Pfau W. Modulation of cytochrome P450 1A1 by food-derived heterocyclic aromatic amines. Toxicology 2004;199:231-40.

13. Crofts F,CosmaGN,Currie D, TaioliE,Toniolo P,GarteSJ.Anovel CYP1A1 gene polymorphism in African-Americans. Carcinogenesis. 1993;14:1729-31.

14. Kawajiri K, Nakachi K, Imai K, Yoshii A, Shinoda N, Watanabe J. Identification of genetically high risk individuals to lung cancer by DNA polymorphisms of the cytochrome P450IA1 gene. FEBS Lett. 1990;263:131-3.

15. Shah, P.P., Saurabh, K., Pant, M.C., Mathur, N., Parmar, D., 2009. Evidence for increased cytochrome P450 1A1 expression in blood lymphocytes of lung cancer patients. Mutat. Res. 670, 74-78. 
16. Zhu X, Wang Z, He J, Wang W, Xue W, Wang Y, Zheng L, Zhu ML. Associations between CYP1A1 rs1048943 A > G and rs4646903 T > C genetic variations and colorectal cancer risk: Proof from 26 case-control studies. Oncotarget. 2016;7(32):51365-51374.

17. Xu L, Wei H. Association between CYP1A1 2454A > G polymorphism and colorectal cancer risk: A meta-analysis. J Cancer Res Ther. 2015 Oct-Dec;11(4):760-4.

18. Liu C, Jiang Z, Deng QX, Zhao YN. Meta-analysis of association studies of CYP1A1 genetic polymorphisms with digestive tract cancer susceptibility in Chinese. Asian Pac J Cancer Prev. 2014;15(11):468995.

19. He XF, Wei W, Liu ZZ, Shen XL, Yang XB, Wang SL, Xie DL. Association between the CYP1A1 T3801C polymorphism and risk of cancer: evidence from 268 case-control studies. Gene. 2014 Jan $25 ; 534(2): 324-44$.

20. Qin J, Zhang JX, Li XP, Wu BQ, Chen GB, He XF. Association between the CYP1A1 A2455G polymorphism and risk of cancer: evidence from 272 case-control studies. Tumour Biol. 2014;35(4):336376.

21. Zheng Y, Wang JJ, Sun L, Li HL. Association between CYP1A1 polymorphism and colorectal cancer risk: a meta-analysis. Mol Biol Rep. 2012 Apr;39(4):3533-40.

22. Jin JQ, Hu YY, Niu YM, Yang GL, Wu YY, Leng WD, Xia LY. CYP1A1 Ile462Val polymorphism contributes to colorectal cancer risk: a meta-analysis. World J Gastroenterol. 2011 Jan 14;17(2):260-6.

23. Swartz MK. The PRISMA statement: a guideline for systematic reviews and meta-analyses. J Pediatr Health Care. 2011;25:1-2.

24. Higgins JP, Thompson SG, Deeks JJ, Altman DG. Measuring inconsistency in meta-analyses. BMJ. 2003;327:557-560.

25. Mantel N, HaenszelW. Statistical aspects of the analysis of data from retrospective studies of disease. J Natl Cancer Inst. 1959;22:719-748.

26. DerSimonian R, Laird N. Meta-analysis in clinical trials. Control Clin Trials 1986;7:177-188.

27. Begg CB, Mazumdar M. Operating characteristics of a rank correlation test for publication bias. Biometrics 1994;50:1088-101.

28. Egger M, Smith DG, Schneider M, et al. Bias in meta-analysis detected by a simple, graphical test. Br Med J 1997;315:629-34.

29. Dual S, Tweedie R. A nonparametric "trim and fill" method of accounting for publication bias in meta-analysis. J Am Stat Assoc 2000;95:89-98.

30. Wakefield J. A Bayesian measure of the probability of false discovery in genetic epidemiology studies. Am J Hum Genet. 2007 Aug;81(2):208-27.

31. Attia J, Thakkinstian A, D'Este C. Meta-analyses of molecular association studies: methodologic lessons for genetic epidemiology. J Clin Epidemiol. 2003;56:297-303.

\section{Hosted file}

Figs. 1-2.pdf available at https://authorea.com/users/374149/articles/491747-evaluation-ofassociation-studies-a-systematic-review-and-meta-analysis-of-cyp1a1-polymorphisms-andcolorectal-cancer-risk

\section{Hosted file}

Table 1-3.pdf available at https://authorea.com/users/374149/articles/491747-evaluation-ofassociation-studies-a-systematic-review-and-meta-analysis-of-cyp1a1-polymorphisms-andcolorectal-cancer-risk 